Wilfrid Laurier University

Scholars Commons @ Laurier

Physics and Computer Science Faculty

Publications

Physics and Computer Science

1988

\title{
Analog Simulation of Coupled Superconducting Weak Links: Locking and Chaos
}

\author{
Wu Binruo \\ Wilfrid Laurier University \\ Yang Zhou-jing \\ Wilfrid Laurier University \\ James A. Blackburn \\ Wilfrid Laurier University, jabjabjab@cogeco.ca \\ S. Vik \\ Wilfrid Laurier University \\ H.J.T. Smith \\ University of Waterloo
}

See next page for additional authors

Follow this and additional works at: https://scholars.wlu.ca/phys_faculty

\section{Recommended Citation}

Binruo, Wu; Zhou-jing, Yang; Blackburn, James A.; Vik, S.; Smith, H.J.T.; and Nerenberg, M.A.H., "Analog Simulation of Coupled Superconducting Weak Links: Locking and Chaos" (1988). Physics and Computer Science Faculty Publications. 25.

https://scholars.wlu.ca/phys_faculty/25

This Article is brought to you for free and open access by the Physics and Computer Science at Scholars Commons @ Laurier. It has been accepted for inclusion in Physics and Computer Science Faculty Publications by an authorized administrator of Scholars Commons @ Laurier. For more information, please contact scholarscommons@wlu.ca. 
Authors

Wu Binruo, Yang Zhou-jing, James A. Blackburn, S. Vik, H.J.T. Smith, and M.A.H. Nerenberg 


\title{
Analog simulation of coupled superconducting weak links: Locking and chaos
}

\author{
Wu Binruo,* Yang Zhou-jing, James A. Blackburn, and S. Vik \\ Department of Physics and Computing, Wilfrid Laurier University, Waterloo, Ontario, Canada \\ H. J. T. Smith \\ Department of Physics, University of Waterloo, Waterloo, Ontario, Canada \\ M. A. H. Nerenberg \\ Department of Applied Mathematics, University of Western Ontario, London, Ontario, Canada
}

(Received 21 August 1987)

\begin{abstract}
An improved analog simulator has been developed for the study of resistively coupled pairs of superconducting weak links. Sets of $I-V$ characteristics, obtained with the aid of this circuit under $\mathrm{dc}$ bias conditions, indicate the dependence of certain features, including the voltage locking zone, on system parameters. Chaos has been observed when ac bias is also present and, for the first time, a state diagram for the system has been determined.
\end{abstract}

\section{INTRODUCTION}

Josephson junctions and superconducting weak links have been the subjects of renewed interest because they exhibit, as do many other nonlinear systems, both chaotic and coherent properties. Junctions are inherently capacitive, and when described by the resistively shunted junction model (RSJC) give rise to differential equations of at least second order in time. As Kautz and Monaco ${ }^{1}$ and others have shown, this in turn leads to predictions of chaotic behavior in the current-voltage characteristics of irradiated devices. Experimental data reported by Iansiti et $a l .^{2}$ appear to confirm such predictions.

A set up in complexity is achieved when two or more such junctions are configured so that they may mutually interact, say, through a parallel resistance or impedance. Lee and Schwarz ${ }^{3,4}$ studied linear arrays of Josephson junctions and reported mutual phase locking in current biased samples. Strenski and Doniach ${ }^{5}$ showed theoretically that when two series connected junctions are shunted by a third Josephson device (as opposed to a passive impedance) the nonirradiated current-biased system would exhibit chaos in certain regions of its $I-V$ characteristic.

Microbridges are simpler than Josephson junctions, in that they are thought to be well described by noncapacitive equivalent circuits (RSJ). A consequence of this is that the governing equation for a current biased weak link is of first order only. As a result, irradiated single links cannot exhibit chaos. However, it was shown by Nerenberg et al. ${ }^{6}$ that a resistively coupled pair of ac biased links constitutes the simplest arrangement of microbridges in which chaos can arise. Recently it was demonstrated $^{7,8}$ that chaos will also appear in a system of three links under dc bias alone. The former study, it should be noted, assumed reactive coupling. This introduces derivatives of higher order into the differential equations, which in turn implies a larger dimensional phase space and thus does not rank the system among the simplest possible. Extensive literature already exists describing the voltage locking which also occurs in configurations of shunted links. ${ }^{9-11}$ In addition, systems involving linear arrays of weak links have been examined $^{12,13}$ and, again, impedance coupling across the links can lead to locking. While it is possible to physically add such coupling shunts to thin-film microbridge samples, as noted in Refs. 9 and 10, quasiparticle leakage will lead to interactions even for directly connected, but closely spaced, links.

The subject of the present work is the resistively coupled pair - the arrangement described in Refs. 6, 9, and 10 -with dc and ac bias currents applied. We report new results concerning both locking and chaos which were obtained by means of an improved electronic ana$\log$ simulation circuit. Of particular interest is the state diagram for the system. Such a map of the chaotic domains in the space defined by the two parameters of the applied ac current (amplitude and frequency) has not appeared previously in the literature.

\section{SYSTEM DESCRIPTION}

The equivalent circuit of the system is illustrated in Fig. 1. Two superconducting weak links are characterized by their critical currents $i_{c 1}$ and $i_{c 2}$, and shunt resistors $R_{1}$ and $R_{2}$. The links are independently biased by ac currents whose amplitudes are $a_{1}$ and $a_{2}$, respectively; they both have a common frequency $\omega$. Fixed bias currents $i_{1}$ and $i_{2}$ are also applied in the directions indicated (series aiding). Finally, coupling is provided by resistor $R_{s}$. Following procedures detailed in Ref. 6 we normalized the current conservation equations by means of the parameters

$$
\begin{aligned}
& R_{0}=R_{1}\left(R_{2}+R_{s}\right) /\left(R_{1}+R_{2}+R_{s}\right), \\
& \alpha=\left(1+R_{s} / R_{2}\right)^{-1} \\
& \delta=\left(1+R_{s} / R_{1}\right) /\left(1+R_{s} / R_{2}\right)
\end{aligned}
$$




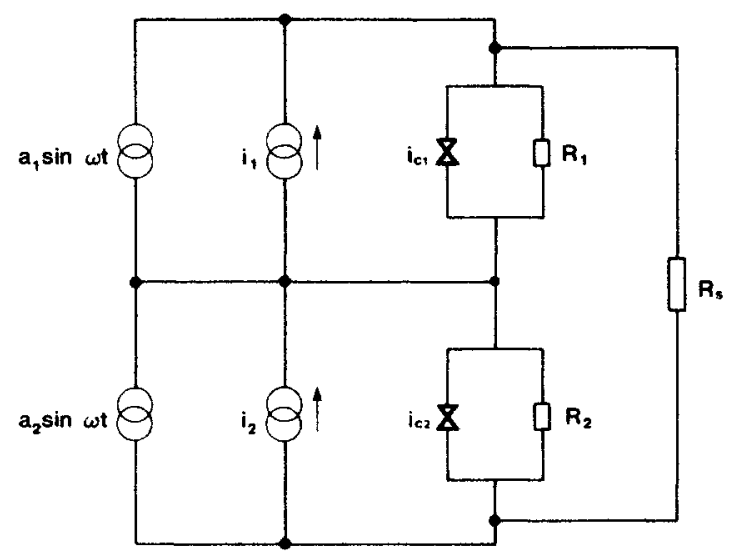

FIG. 1. Weak-link pair with series-aiding dc bias, applied ac bias, and coupling resistors.

Notice that from these equations one may infer $R_{1}, R_{2}, R_{s}$ from any specified set $\left(\alpha, \delta, R_{0}\right)$. Time will be expressed in terms of the reciprocal of the frequency

$$
\omega_{0}=\frac{2 e}{\hbar} \bar{i}_{c} R_{0}
$$

and all currents will be scaled to the average $\left(\bar{i}_{c}\right)$ of the two critical currents $i_{c 1}$ and $i_{c 2}$. With these definitions, the equations describing the coupled links become

$\dot{\phi}_{1}=I_{1}-I_{c 1} \sin \left(\phi_{1}\right)-\alpha\left(I_{2}-I_{c 2} \sin \phi_{2}\right)+b_{1} \sin (\omega t)$,

$\dot{\phi}_{2}=\delta\left(I_{2}-I_{c 2} \sin \phi_{2}\right)-\alpha\left(I_{1}-I_{c 1} \sin \phi_{1}\right)+b_{2} \sin (\omega t)$.
Dots indicate derivatives with respect to normalized time, and the ac amplitudes are given by

$$
\begin{aligned}
& b_{1}=\left(a_{1}-\alpha a_{2}\right) / \overline{i_{c}}, \\
& b_{2}=\left(\delta a_{2}-\alpha a_{1}\right) / \overline{i_{c}} .
\end{aligned}
$$

The pair of coupled first-order differential equations (1) and (2) fully characterize the physical system shown in Fig. 1. A total of nine independent parameters are involved, namely, $\alpha, \delta, I_{1}, I_{2}, I_{c 1}, I_{c 2}, b_{1}, b_{2}$, and $\omega$; normalization through time scaling has reduced the original number of variables by 1 . As noted in our earlier paper, ${ }^{6}$ a comprehensive exploration of the solutions of Eqs. (1) and (2) is impractical. Instead, judicious choices must be made for most of the parameters, while the remaining ones are varied in the hope of uncovering all significant modes which the system is capable of exhibiting. A further limitation on progress towards more complete examinations of the behavior throughout major portions of parameter space is imposed by the substantial amounts of machine time which are consumed in typical numerical solutions of the coupled differential equations. An attractive alternative approach is to devise an electronic circuit which can simulate the original equations.

Over the past decade a number of circuits have been described which are capable of accurately simulating Josephson junctions. ${ }^{14-19}$ Electronic simulators have also been designed for Josephson-junction SQUID interferometers ${ }^{20,21}$ and have proved to be very useful in studying chaos in ac biased capacitive junctions. ${ }^{22-24}$ The only application of analog simulation to the coupled

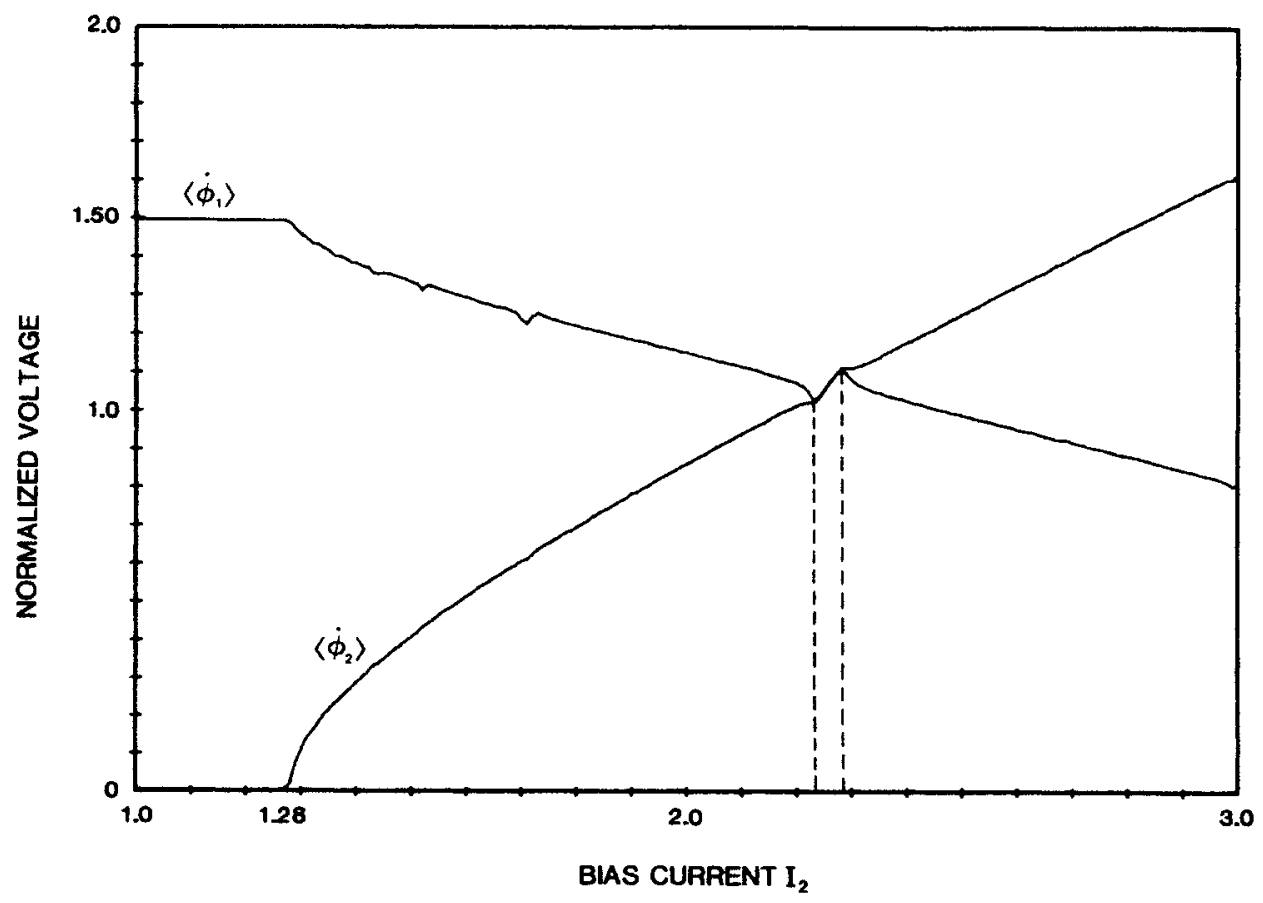

FIG. 2. Time-averaged link voltages as a function of the swept bias current on link No. 2. Parameters are $\alpha=0.2, \delta=\frac{2}{3}$, $I_{1}=2.0, I_{c 1}=1.2, I_{c 2}=0.8$, and $b=0$. 


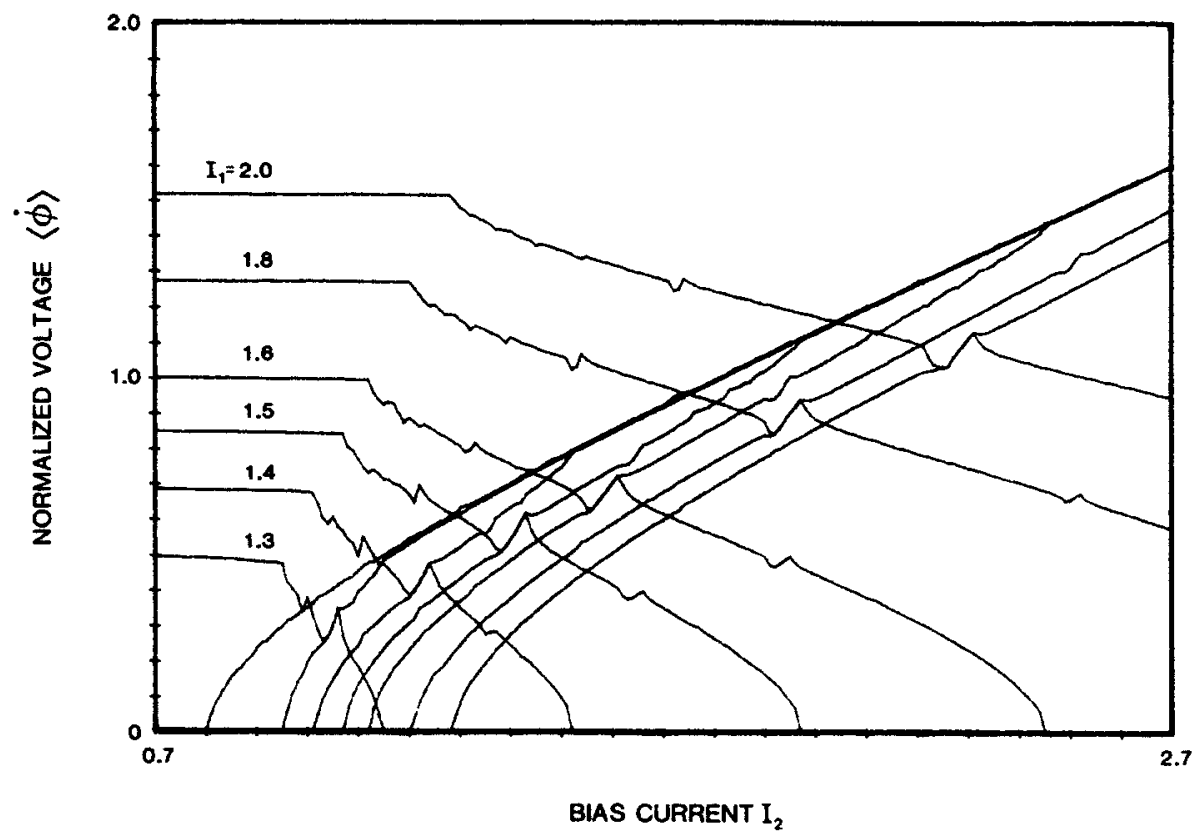

FIG. 3. Set of characteristic curves, as in Fig. 1, but with fixed bias on link No. 1 varying between 1.3 and 2.0 . The parabolic asymptote is obtained when $I_{1}=0$.

weak-link configuration appears to be that of Escudero and Smith, ${ }^{25}$ who were able to confirm some of the locking results of Ref. 9. For the present work an improved version of their circuit has been developed; a complete description and schematic are to be found in the Appendix. Data collection and analysis were performed by a desk-top computer equipped with two channels of analog-to-digital conversions. Appropriate voltages corresponding to $\phi$ or $\dot{\phi}$ were acquired and time averaged, as needed. In addition, digital-to-analog channels were available for control of independent variables in each experiment.

\section{LOCKING WITHOUT ac BIAS

$$
\left(b_{1}=b_{2}=0\right)
$$

As an initial test of the analog simulator, circuit components (see Appendix) were selected to correspond to $\alpha=0.2, \delta=\frac{2}{3}, I_{c 1}=1.2, I_{c 2}=0.8$, and $I_{1}=2.0$. $I_{2}$ was

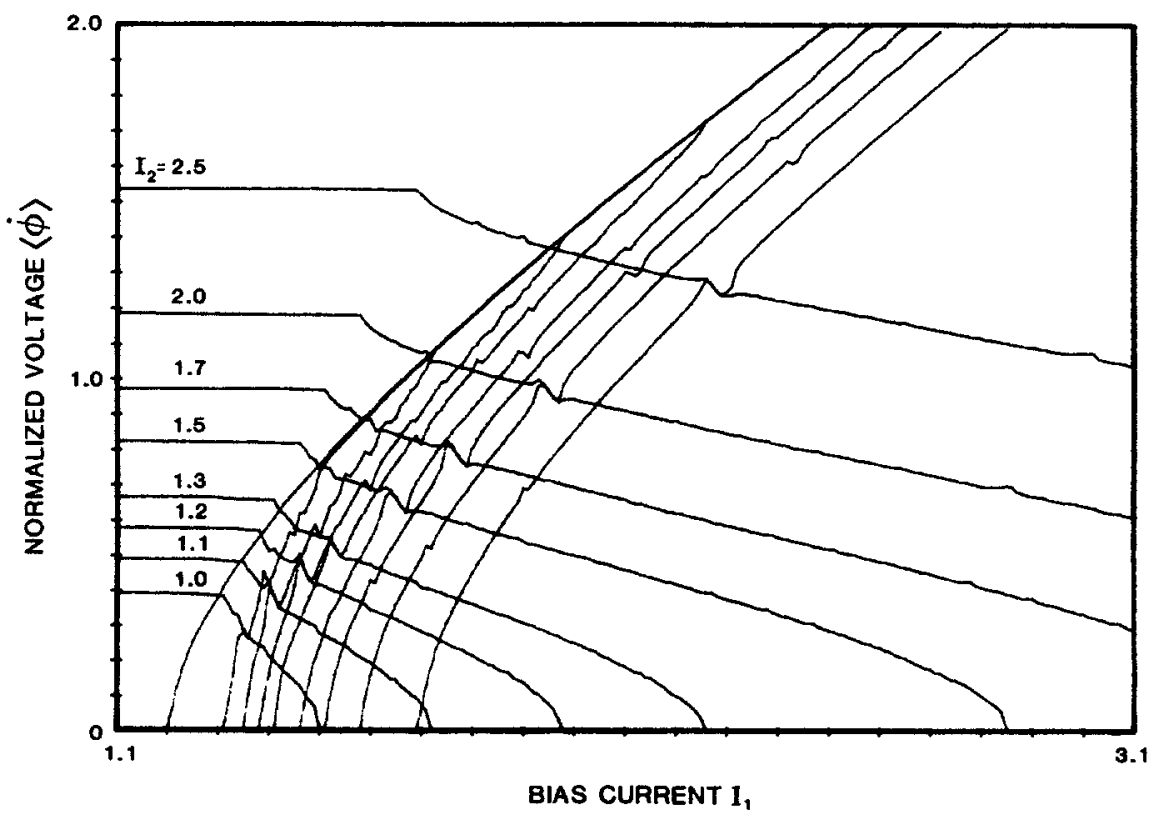

FIG. 4. Characteristic curves, similar to Fig. 3, but with $I_{2}$ fixed at selected values and $I_{1}$ varying. 


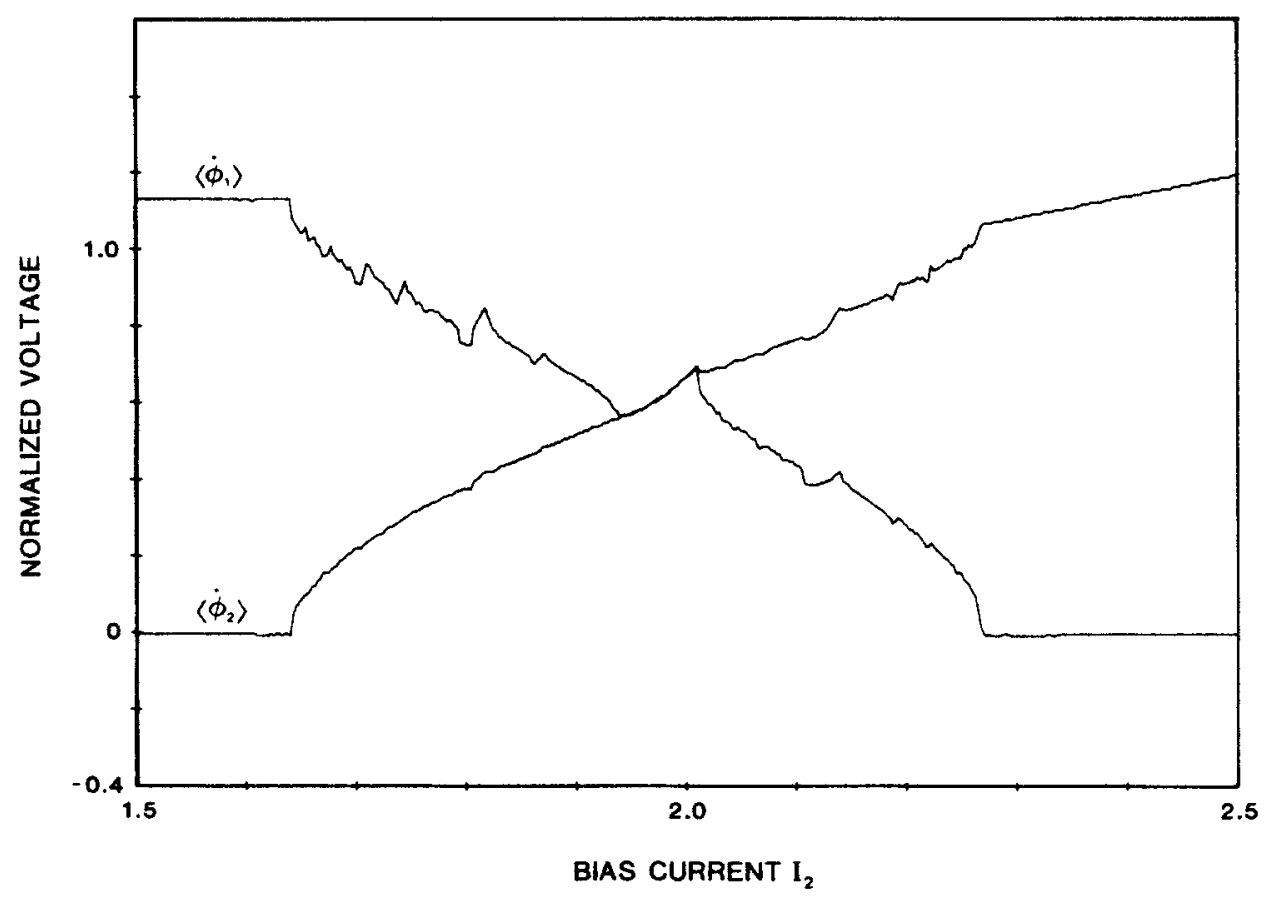

FIG. 5. Link voltages vs sweeping current $I_{2}$ for a stronger coupling coefficient, $\alpha=0.4$. Other parameters are the same as for Fig. 2.

swept through the range $1.0-3.0$ and the time-averaged link voltages (denoted by \langle\rangle ) were plotted as functions of $I_{2}$. The results are presented in Fig. 2, where a number of harmonic locking regions, with $\left\langle V_{1}\right\rangle=n\left\langle V_{2}\right\rangle$, are evident in addition to the central locking zone which extends approximately from $I_{2}=2.2$ to 2.3.

A set of characteristics was obtained by running the simulator with successively larger values of $I_{1}$, beginning at 1.3. As can be seen in Fig. 3, the central locking zones are steadily shifted toward larger $I_{2}$ and, as well, the width of the zones tends to increase somewhat. Also plotted in this figure is the $\left\langle V_{2}\right\rangle$ versus $I_{2}$ characteristic when $I_{1}$ is well below the critical current of link No. 1 (i.e., $I_{c 1}$ ). This curve acts an asymptote towards which all other $\left\langle V_{2}\right\rangle-I_{2}$ characteristics are attracted at suitably large $I_{2}$. Similar results are obtained when $I_{2}$ is fixed

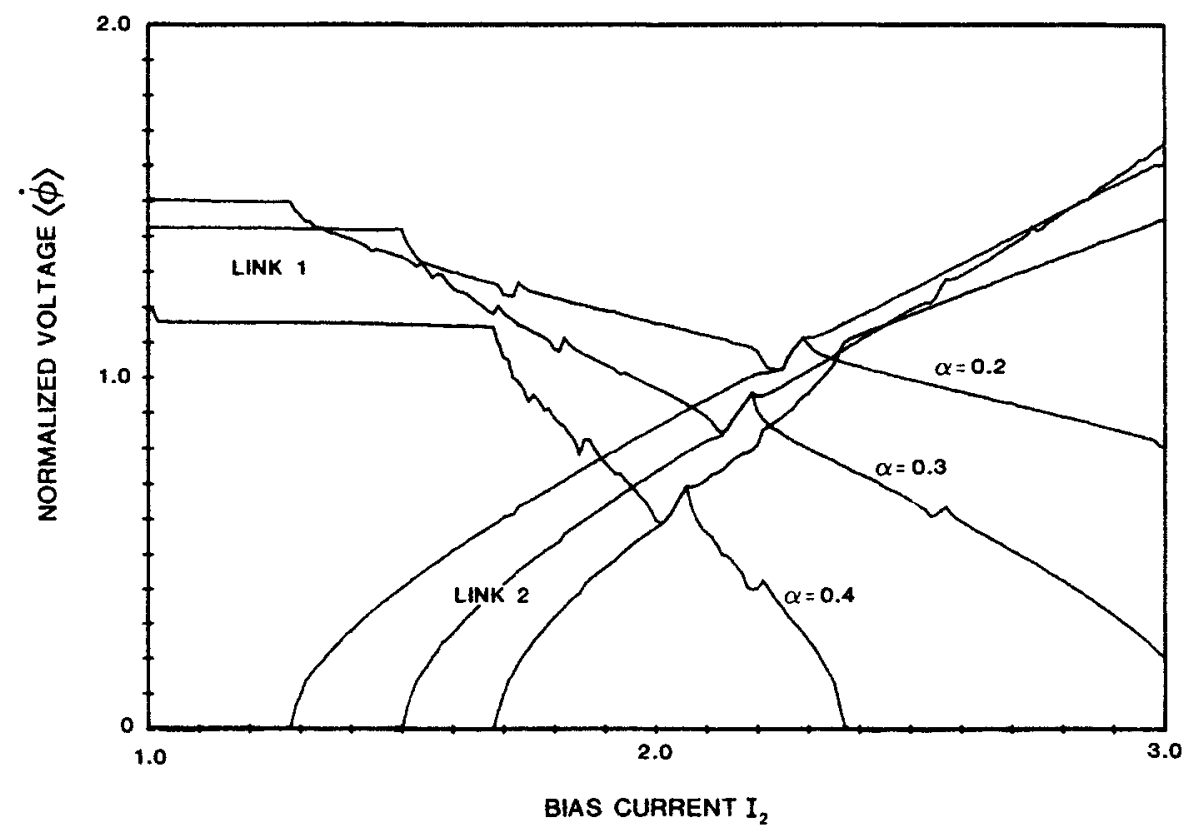

FIG. 6. Set of characteristics, similar to Fig. 4, but with three choices for $\alpha$. 
and $I_{1}$ is scanned, except for the fact that the direction of the locking region is reversed. This is apparent in Fig. 4 and is due to the tendency of the central locking zone to align itself along the characteristic belonging to the link with the smaller value of shunt resistance (link No. 2 in these particular examples).

We next consider the effect on the characteristics of variations in the value of the coupling coefficient. Figure 5 shows results obtained from the analog simulator for parameter values identical to those of Fig. 2, with the exception of $\alpha$, which is now doubled to 0.4 . The central locking region is no longer linear. Such curvature has also been reported by $\mathrm{Dai}$ and $\mathrm{Kao}^{11}$ and McDonald, ${ }^{13}$ but in both these cases the nonlinearity is associated with reactive coupling. In contrast, the curvature here is due entirely to the strength of the purely resistive interaction between the links. Figure 6 illustrates the effect on the characteristics of progressively increasing the value of $\alpha$. It is possible to explain several features in the above results by means of an approximate analysis of the nonlinear equations governing the system.

\section{A. Critical current of link No. 2}

For a single nonirradiated link, the superconducting phase satisfies

$$
\dot{\phi}=K\left[i_{0}-i_{c} \sin (\phi)\right],
$$

where $i_{0}$ is the applied dc bias current. By elementary methods one may obtain from this equation $\langle\dot{\phi}\rangle=K\left(i_{0}^{2}-i_{c}^{2}\right)^{1 / 2}$. Consider the case (Figs. 2 and 3 ) where $I_{1}$ is fixed and $I_{2}$ is scanned. Let us fix our attention momentarily on the $\left\langle V_{2}\right\rangle$ versus $I_{2}$ curves. Clearly for each such curve a critical current $I_{c 2}^{*}$ exists below which $\left(V_{2}\right)$ is zero. This is not the same as the link's intrinsic critical current because of the effect of interactions from link No. 1, which is already biased and oscillating. Time averaging Eq. (2) and noting that $\left\langle\dot{\phi}_{2}\right\rangle=0$, gives at the effective critical current

$$
I_{2}=I_{c 2}+\frac{\alpha}{\delta}\left\langle I_{1}-I_{c 1} \sin \phi_{1}\right\rangle
$$

where we have dropped terms which average to zero, at least in a first approximation. The bracketed term may be determined, again to lowest order in $\alpha$, from Eq. (1) with the result

$$
I_{c 2}^{*}=I_{c 2}+(\alpha / \delta)\left(I_{1}^{2}-I_{c 1}^{2}\right)^{1 / 2} .
$$

We have used the fact that $I_{2}$ is identical to $I_{c 2}^{*}$ at this particular bias point. A more formal perturbation calculation would lead to an identical result. ${ }^{9}$

If we choose, for example, $\alpha=0.2, \delta=\frac{2}{3}, I_{1}=2.0$, $I_{c 1}=1.2$, and $I_{c 2}=0.8$, then Eq. (5) predicts that $I_{c 2}^{*}=1.28$. This value is in excellent agreement with the simulation results shown in Fig. 2. By a similar argument, when $I_{2}$ is fixed and $I_{1}$ is scanned, we find

$$
I_{c 1}^{*}=I_{c 1}+\alpha\left(I_{2}^{2}-I_{c 2}^{2}\right)^{1 / 2} \text {. }
$$

\section{B. Constant voltage of link No. 1}

Turning now to the $\left\langle V_{1}\right\rangle$ versus $I_{2}$ curves in Figs. 2 and 3 , we observe that when $I_{2}<I_{c 2}^{*},\left\langle V_{1}\right\rangle$ remains at a constant level. From Eqs. (1) and (2) we obtain

$$
\dot{\phi}_{1}=\left(1-\alpha^{2} / \delta\right)\left[I_{1}-I_{c 1} \sin \left(\phi_{1}\right)\right]-(\alpha / \delta) \dot{\phi}_{2} \text {. }
$$

The final term may be dropped because $I_{2}$ is below its effective critical value over this interval and so $\dot{\phi}_{2}$ averages to zero. As noted in connection with $\mathrm{Eq}$. (3), this then leads to the result

$$
\left\langle\dot{\phi}_{1}\right\rangle=\left(1-\alpha^{2} / \delta\right)\left(I_{1}^{2}-I_{c 1}^{2}\right)^{1 / 2} .
$$

Returning to the parameter values selected for Fig. 2, this equation predicts a constant level of 1.504 in good agreement with the analog simulation data.

\section{End points of locking zone}

If we define

$$
\begin{aligned}
& \zeta_{1}=I_{1}-\alpha I_{2}, \quad \eta_{1}=\alpha I_{c 2}, \quad \Delta_{1}=I_{c 1}-\eta_{1}, \\
& \xi_{2}=\delta I_{2}-\alpha I_{1}, \quad \eta_{2}=\alpha I_{c 1}, \quad \Delta_{2}=\delta I_{c 2}-\eta_{2},
\end{aligned}
$$

then Eqs. (1) and (2) are expressible in the form

$$
\begin{aligned}
& \dot{\phi}_{1}=\xi_{1}-\left[2 \eta_{1} \cos \left(\frac{\phi_{1}+\phi_{2}}{2}\right) \sin \left(\frac{\phi_{1}-\phi_{2}}{2}\right)+\Delta_{1} \sin \phi_{1}\right], \\
& \dot{\phi}_{2}=\xi_{2}-\left[2 \eta_{2} \cos \left[\frac{\phi_{2}+\phi_{1}}{2}\right] \sin \left[\frac{\phi_{2}-\phi_{1}}{2}\right]+\Delta_{2} \sin \phi_{2}\right] .
\end{aligned}
$$

The modulation factors involving sums and differences of the individual phases vanish in the locking zone. This condition can occur under either of two conditions: (a) $\phi_{1}-\phi_{2}=(2 n+1) \pi$ or (b) $\phi_{1}-\phi_{2}=2 n \pi$. With the first condition, Eqs. (9) and (10) become

$$
\begin{aligned}
& \dot{\phi}_{1}=\zeta_{1}-\left(2 \eta_{1}+\Delta_{1}\right) \sin \left(\phi_{1}\right), \\
& \dot{\phi}_{2}=\xi_{2}-\left(2 \eta_{2}+\Delta_{2}\right) \sin \left(\phi_{2}\right) .
\end{aligned}
$$

The second condition yields

$$
\begin{aligned}
& \dot{\phi}_{1}=\xi_{1}-\Delta_{1} \sin \left(\phi_{1}\right), \\
& \dot{\phi}_{2}=\zeta_{2}-\Delta_{2} \sin \left(\phi_{2}\right) .
\end{aligned}
$$

As noted earlier in connection with Eq. (3), time averages may be written down directly. Asserting the additional requirement for locking, $\left\langle\dot{\phi}_{1}\right\rangle=\left\langle\dot{\phi}_{2}\right\rangle$, we obtain the following two expressions originating from conditions (a) and (b), respectively:

$$
\begin{aligned}
& {\left[\zeta_{1}^{2}-\left(2 \eta_{1}+\Delta_{1}\right)^{2}\right]^{1 / 2}=\left[\zeta_{2}^{2}-\left(2 \eta_{2}+\Delta_{2}\right)^{2}\right]^{1 / 2},} \\
& \left(\zeta_{1}^{2}-\Delta_{1}^{2}\right)^{1 / 2}=\left(\zeta_{2}^{2}-\Delta_{2}^{2}\right)^{1 / 2} .
\end{aligned}
$$

From (15) we may solve for $I_{2}$, 


$$
I_{2 a}=\frac{-\alpha I_{1}(1-\delta)+\left\{\alpha^{2} I_{1}^{2}(1-\delta)^{2}+\left(\delta^{2}-\alpha^{2}\right)\left[\left(1-\alpha^{2}\right) I_{1}^{2}-\left(I_{c 1}+\alpha I_{c 2}\right)^{2}+\left(\alpha I_{c 1}+\delta I_{c 2}\right)^{2}\right]\right\}^{1 / 2}}{\delta^{2}-\alpha^{2}}
$$

Similarly, from (16) we obtain

$$
I_{2 b}=\frac{-\alpha I_{1}(1-\delta)+\left\{\alpha^{2} I_{1}^{2}(1-\delta)^{2}+\left(\delta^{2}-\alpha^{2}\right)\left[\left(1-\alpha^{2}\right) I_{1}^{2}-\left(I_{c 1}-\alpha I_{c 2}\right)^{2}+\left(\delta I_{c 2}-\alpha I_{c 1}\right)^{2}\right]\right\}^{1 / 2}}{\delta^{2}-\alpha^{2}}
$$

Equations (17) and (18) determine the end points of the locking zone when the bias is swept on link No. 2 . For the example presented in Fig. 2, substitution of parameter values leads to predictions that $I_{2 a}=2.22$ and $I_{2 b}=2.34$. From the analog simulator we find $I_{2 a}=2.22$ and $I_{2 b}=2.28$. Numerical solutions of the differential equations, as described in Ref. 9, yield $I_{2 a}=2.18$ and $I_{2 b}=2.24$. A less intuitive approach ${ }^{26}$ gives by perturbation theory $I_{2 a}=2.17$ and $I_{2 b}=2.25$. Equations (17) and (18) are thus useful approximate predictors of the location of the locking region. As a final comment, we note that Dai and $\mathrm{KaO}^{11}$ also derive expressions for the minimum and maximum currents under which locking would occur, but for resonantly coupled identical junctions.

$$
\text { IV. CHAOS }\left(b_{1}=b_{2} \neq 0\right)
$$

As was shown in Ref. 6, chaos arises in this system of coupled links, under appropriate conditions, when ac bias is applied. In that work all parameters were fixed at specified values, except for the ac amplitude, which was varied up to about 1.5. At the selected ac frequency of
0.4 it was found that locking extended from $b$ near zero to about 0.37 . This was followed at increasing amplitudes by alternating regions of chaos and periodicity. These results were obtained from numerical solutions of the coupled differential equations and suffered from the limitations, already mentioned, which are imposed by the substantial amounts of computer time consumed in each run. The analog simulator, in contrast, allows very large amounts of data to be generated efficiently and economically. Thus it was possible to undertake a more extensive investigation of the system dynamics. It should be noted, however, that Lyapunov coefficients cannot be extracted directly from the simulator data, although one might hope that the highly interactive technique of Wolf et al. ${ }^{27}$ could prove useful in such a situation.

To begin, components (see Appendix) were selected which would correspond to $\alpha=0.2, \delta=\frac{2}{3}, I_{1}=2.0$, $I_{c 1}=1.2, I_{2}=2.2, I_{c 2}=0.8$, and $\omega=0.4$. The ac amplitude $b$ was adjusted to values over the interval $0.0-2.0$ and a plot of $\left\langle V_{1}\right)$ and $\left(V_{2}\right\rangle$ versus $b$ was constructed; this is shown in Fig. 7. It will be noted that this result from the analog simulator is in very good agreement

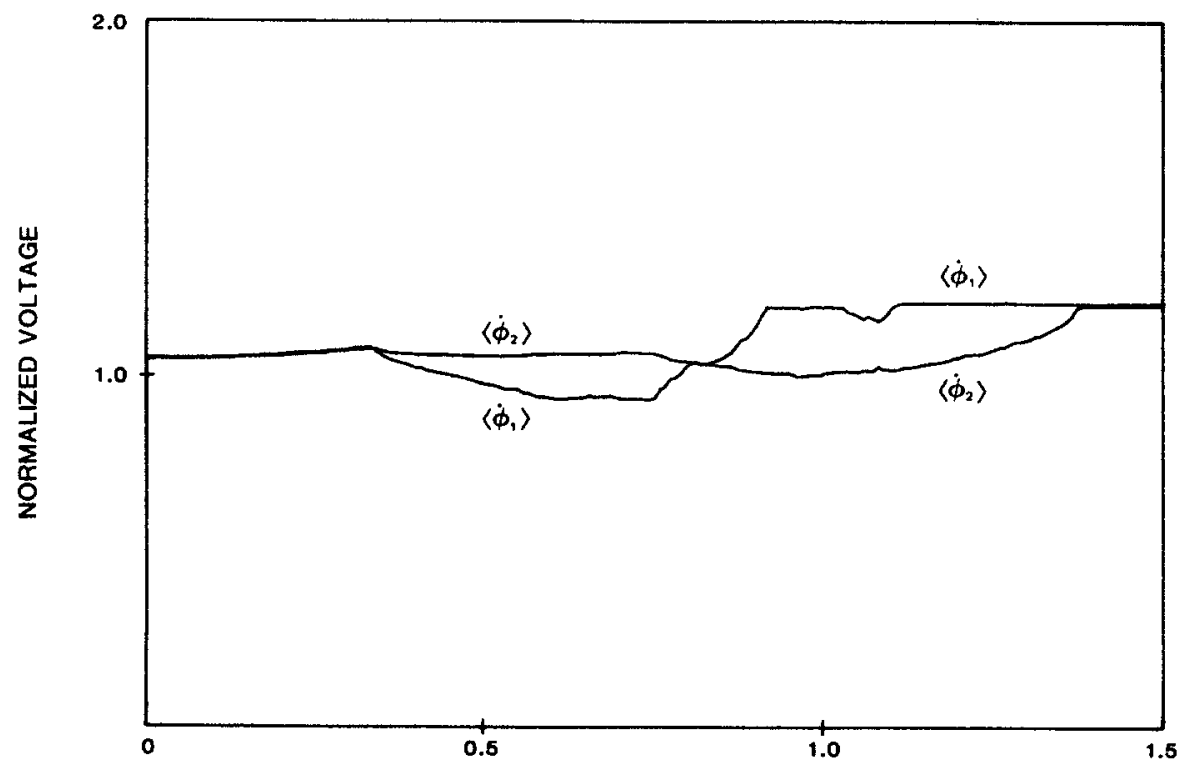

AC AMPLITUDE b

FIG. 7. Time-averaged link voltages as a function of amplitude of the applied ac bias for $\omega=0.4$. Parameter values $\alpha=0.2$, $\delta=\frac{2}{3}, I_{1}=2.0, I_{2}=2.20, I_{c l}=1.2$, and $I_{c 2}=0.8$ were selected to place the system in the locking region of Fig. 2 when $b$ is small. 
with the digital computations illustrated in Fig. 2 of Ref. 6. It is also possible by extracting appropriate signals from the simulator circuit to display Poincare plots which accumulate in real time. This is done by sampling the link voltages once in every cycle of the ac drive. By this means we were able to confirm the appearance of chaotic regions within the interval specified above for $b$. It should be remarked in this connection that for ac amplitudes below the first splitting point (when the initial locking is first broken), the Poincare plots take the form of a one-dimensional curve, indicating that the junctions are free running with respect to the ac drive signal. The precise shape of such a curve in $\sin \left(\phi_{1}\right)$-sin $\left(\phi_{2}\right)$ space is in effect a remapping of the profile of the link voltages. Periodic behavior manifests itself as a stable $n$-point display, while chaos is indicated by the typical pattern of a strange attractor. These features are illustrated by the plots in Fig. 8.

By repeating the above procedure for different ac drive frequencies and assessing the Poincare plots with the aid of the method of standard deviations described in Ref. 28, a state diagram for the system was constructed. Because data acquisition and parameter selection were under the control of a desk-top computer, the process (a)

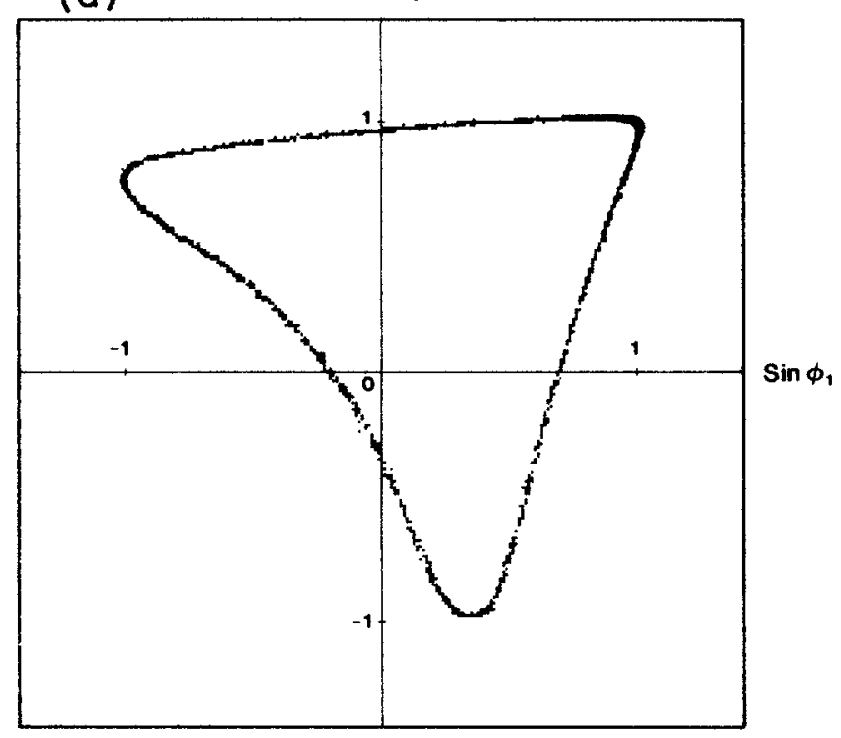

(b)

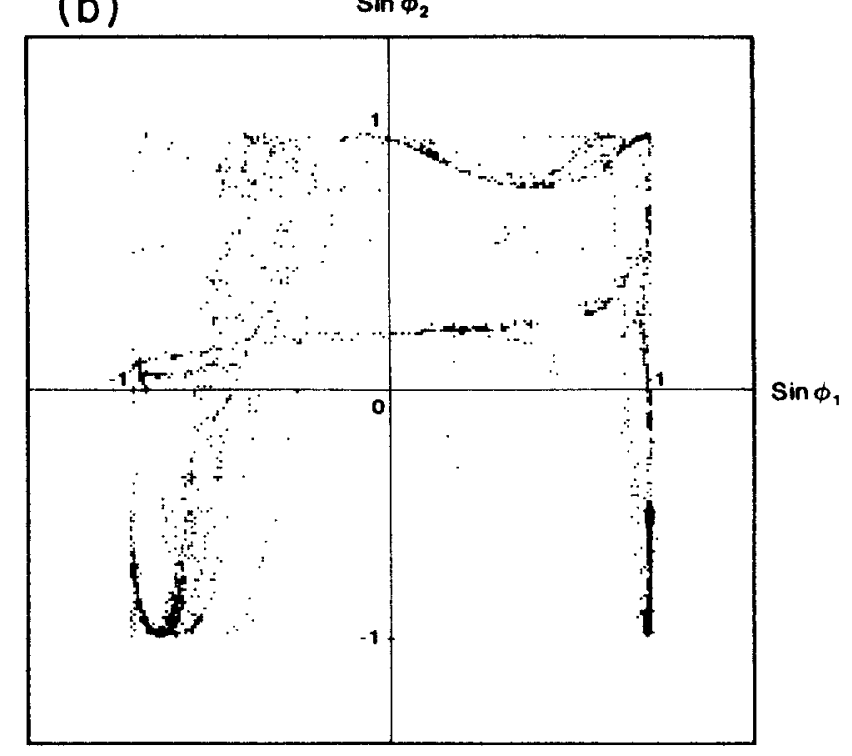

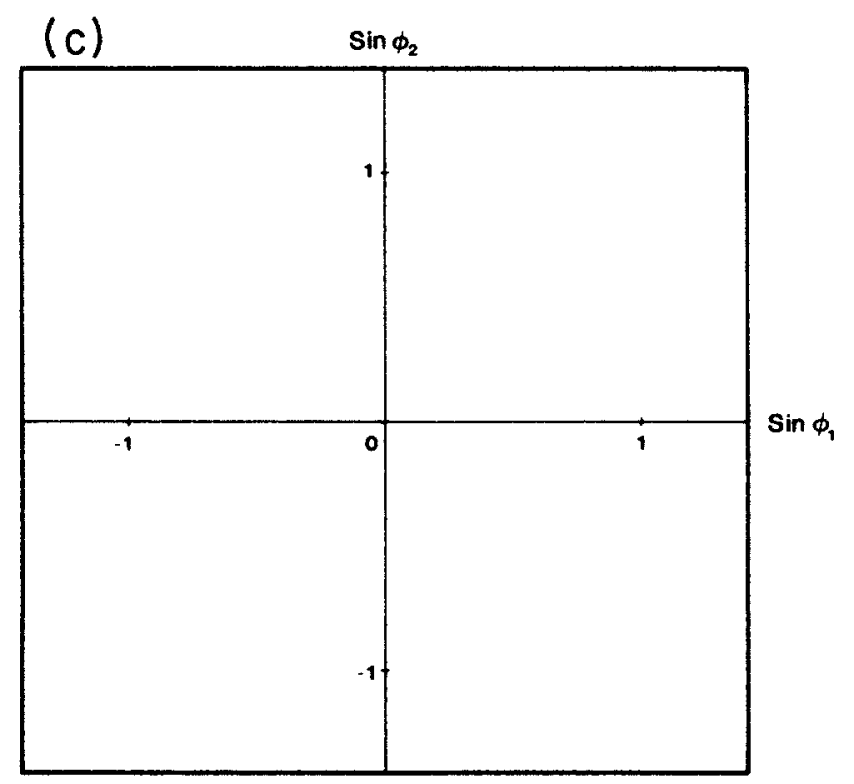

FIG. 8. Poincaré plots derived by sampling $\sin \left(\phi_{1}\right)$ and $\sin \left(\phi_{2}\right)$ once in each cycle of the applied ac bias. Parameters common to the three diagrams are $\alpha=0.2, \delta=\frac{2}{3}, I_{1}=2.0, I_{2}=2.20, I_{c 1}=1.2, I_{c 2}=0.8$, and $\omega=0.4$. (a) $b=0.1$; here the links are locked but free running with respect to the applied ac bias. (b) $b=0.84$, chaos. (c) $b=0.88$, period 2 solution. 


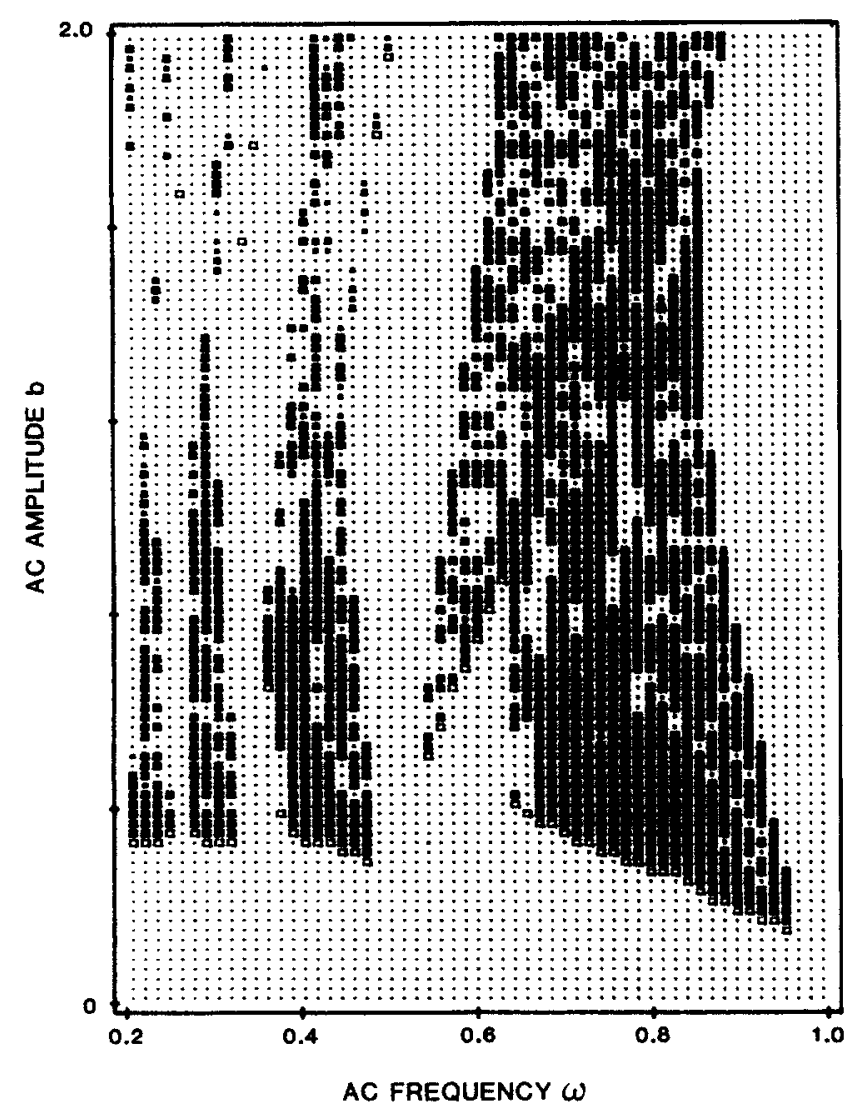

FIG. 9. State diagram for the coupled weak-link pair. Chaotic regions are indicated by black squares; dots indicate periodic or multiply periodic behavior.

was highly automated. The results are represented in Fig. 9 and reveal where, in the space defined by the amplitude and frequency of the ac drive, chaos occurs. The pattern is in many ways similar to the state diagram given in Ref. 28 for a driven pendulum (or, equivalently, an ac biased capacitive Josephson junction). In particular, it is apparent that distinct chaotic bands exist within which lie complex subregions of periodic behavior. However, in contrast to the driven pendulum diagram, these bands are vertically aligned so that for certain frequency intervals the dynamics seem to be chaos free for all ac amplitudes. For the parameters selected here, the bands are approximately located around normalized frequencies of $\frac{1}{2}, \frac{1}{3}$, and $\frac{1}{4}$. Such bands also are found for the pendulum but they are oriented diagonally in the $b-\omega$ plane. As yet no theoretical basis has been found for this feature of state diagrams. At frequencies somewhat lower than those included in Fig. 9, the first onset of chaos was found to occur at smaller ac amplitudes $(b<0.2)$. Finally we note that in agreement with the conclusions of Ref. 6, which were based on highprecision numerical simulations, the route to chaos does not appear to be of the period-doubling type. Rather, at chaotic boundaries abrupt transitions are seen to more complex states of high period number.

\section{CONCLUSIONS}

An improved analog simulation circuit has been used to study the behavior of a coupled pair of current biased superconducting weak links. The performance of this circuit has been tested by comparing current-voltage characteristics with previously published numerical results for this system. Good agreement is found, particularly with respect to the locking zones. New data reveal in more detail the dependence of the locking on several relevant parameters, and in particular, on the coupling coefficient. Chaos has also been observed with the simulator and a complete state diagram for the system has been determined. An interesting feature of this map is the apparent absence of chaos for certain intervals in the frequency of the applied ac bias.

\section{ACKNOWLEDGMENT}

Funding for this research was provided by the Natural Sciences and Engineering Research Council of Canada.

\section{APPENDIX}

The circuit used by Escudero and Smith ${ }^{25}$ to model a pair of coupled weak links was based on the design of Henry et al. ${ }^{19}$ The fundamental operating principles of these circuits, which are modified phase-locked loops, have been adequately discussed in these references and will not be repeated here. Two points concerning the designs do, however, deserve comment. In both Refs. 17 and 25 the output of a mixer (Analog Devices 532) was fed to an $R C$ low-pass filter. This essential step was necessary to remove higher harmonics of the voltagecontrolled oscillator (VCO) (Analog Devices 537) square-wave output, as well as unwanted products from the mixing operation itself. Unfortunately, the $R C$ filter also induces an undesirable phase shift into the feedback path of the summing amplifier. A second performance limitation is introduced by the requirement of a highly stable reference oscillator.

The complete schematic of the new analog simulator is shown in Fig. 10. The overall configuration [Fig. $10(a)]$ remains as in the above-mentioned references, but a significantly different approach has been taken with respect to the VCO module [Fig. 10(b)], which no longer employs an AD537 and does not need an external oscillator. In addition, the $R C$ filter and its inherent problems have been eliminated in favor of a matched-diode sine converter of the type (CA3019) often found in precision signal generators.

The operation of the VCO module may be summarized as follows. The voltage comparator $U_{1}$ is used as a zero-crossing detector. Its square-wave output controls the analog switch $\left(U_{2}\right)$, which alternately delivers $\pm 8 \mathrm{~V}$ to one input of the multiplier $U_{3}$, while the other input receives the control voltage $V_{c}$. The output of the multiplier is thus a square wave whose amplitude is proportional to the control voltage. Let us assume for the moment that $V_{c}>0$ and that the outputs of $U_{1}$ and $U_{2}$ are positive. This will, through $U_{7}$ and $U_{8}$, select channel 1 on the second analog switch $\left(U_{6}\right)$, and pass the integrat- 

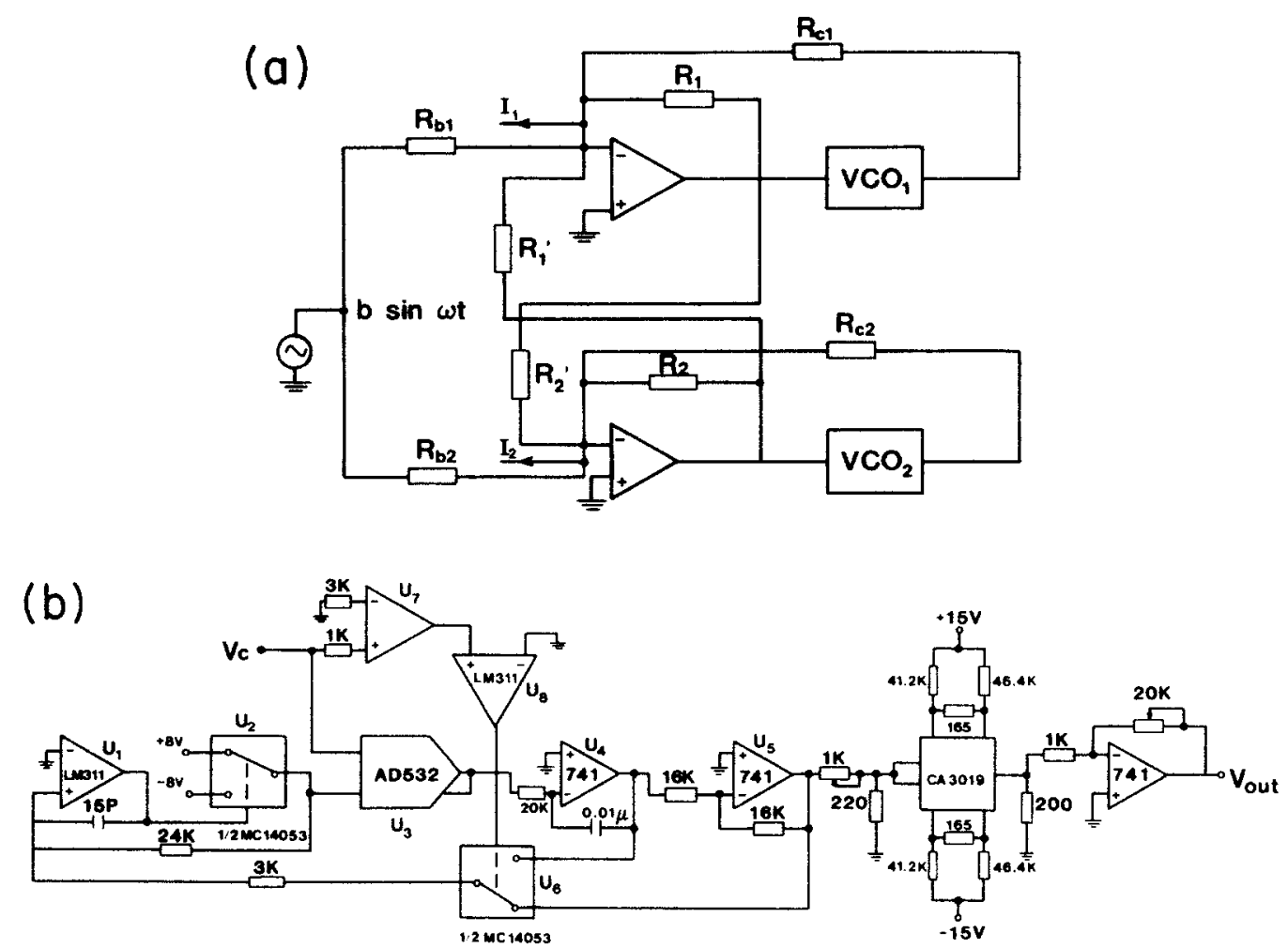

FIG. 10. Simplified schematics of (a) the overall simulation circuit and (b) the elements of the voltage-controlled oscillator.

ed square wave-a linear ramp with negative slopefrom $U_{4}$ to $U_{1}$ via resistors $R_{2}$ and $R_{3}$. When this reaches $-\left(R_{2} / R_{3}\right) 8 \mathrm{~V}, U_{1}$ will switch and select $-8 \mathrm{~V}$ for the analog multiplier input. The integrator output will now be a ramp with positive slope until $+\left(R_{2} / R_{3}\right) 8$ $V$ is reached, at which point the zero-crossing detector will switch again. Thus the input of $U_{4}$ will be a sawtooth waveform, which is converted to a sine wave by the diode array, and buffered by a final operational amplifier. The second analog switch $\left(U_{6}\right)$ is necessary to accommodate negative control voltages $V_{c}$ by feeding inverted ramps from $U_{5}$ to $U_{1}$.
As noted by Escudero and Smith, ${ }^{25}$ this circuit will simulate the coupled equations provided the various resistors are selected according to the following relations:

$$
\begin{aligned}
& R_{1}=r_{0}\left(1-\alpha^{2} / \delta\right), \quad R_{2}=r_{0} \delta\left(1-\alpha^{2} / \delta\right), \\
& R_{1}^{\prime}=R_{2}^{\prime}=r_{0}(\delta / \alpha)\left(1-\alpha^{2} / \delta\right) \\
& R_{b 1}(\alpha+\delta)=R_{b 2}(1+\alpha)
\end{aligned}
$$

As before $R_{c 1}$ and $R_{c 2}$ set the equivalent of the link critical currents and the scaling resistor $r$ was chosen to be $10 k \Omega$.
*Permanent address: Chengdu Institute of Radio Engineering, Chengdu, Sichuan, People's Republic of China.

†Permanent address: University of Science and Technology of China, Hefei, Anhui, People's Republic of China.

${ }^{\text {'}}$ R. L. Kautz and R. Monaco, J. Appl. Phys. 57, 875 (1985).

${ }^{2}$ M. Iansiti, Q. Hu, R. M. Westervelt, and M. Tinkham, Phys. Rev. Lett. 55, 746 (1985).

${ }^{3}$ G. S. Lee and S. E. Schwarz, J. Appl. Phys. 55, 1035 (1984).

${ }^{4}$ G. S. Lee and S. E. Schwarz, J. Appl. Phys. 60, 465 (1986).

${ }^{5}$ P. N. Strenski and S. Doniach, J. Appl. Phys. 57, 867 (1985).

${ }^{6}$ M. A. H. Nerenberg, J. A. Blackburn, and S. Vik, Phys. Rev. B 30, 5084 (1984).

${ }^{7}$ P. Hadley and M. Beasley, Appl. Phys. Lett. 50, 621 (1987).

${ }^{8}$ M. A. H. Nerenberg, J. H. Baskey, and J. A. Blackburn, Phys. Rev. B 36, 8333 (1987).
${ }^{9}$ M. A. H. Nerenberg, J. A. Blackburn, and D. W. Jillie, Phys. Rev. B 21, 118 (1980).

${ }^{10}$ D. W. Jillie, M. A. H. Nerenberg, and J. A. Blackburn, Phys. Rev. B 21, 125 (1980).

${ }^{11}$ Y.-D. Dai and Y. H. Kao, J. Appl. Phys. 52, 4135 (1981).

${ }^{12}$ M. A. H. Nerenberg and J. A. Blackburn, Phys. Rev. B 23, 1149 (1981).

${ }^{13}$ D. G. McDonald, J. Appl. Phys. 60, 3247 (1986).

${ }^{14}$ C. K. Bak and N. F. Pedersen, Appl. Phys. Lett. 22, 149 (1973).

15J. H. Magerlein, Rev. Sci. Instrum. 49, 486 (1978).

${ }^{16}$ A. Yagi and I. Kurosawa, Rev. Sci. Instrum. 51, 14 (1980).

${ }^{17}$ R. W. Henry, D. E. Prober, and A. Davidson, Am. J. Phys. 49, 1035 (1981).

${ }^{18}$ D. E. Prober, S. E. Slusky, R. W. Henry, and L. D. Jackel, J. 
Appl. Phys. 52, 4145 (1981).

${ }^{19}$ D. G. Jablonski, J. Appl. Phys. 53, 7458 (1982).

${ }^{20}$ D. B. Tuckerman, Rev. Sci. Instrum. 49, 835 (1978).

${ }^{21}$ R. W. Henry and D. E. Prober, Rev. Sci. Instrum. 52, 902 (1981).

${ }^{22}$ Y. H. Kao, J. C. Huang, and Y. S. Gou, J. Low Temp. Phys. 63, $287(1986)$.

${ }^{23}$ Y. H. Kao, J. C. Huang, and Y. S. Gou, Phys. Rev. A 34, 1628 (1986).

${ }^{24}$ D. D'Humieres, M. R. Beasley, B. A. Huberman, and A. Lib- chaber, Phys. Rev. A 26, 3483 (1982).

${ }^{25}$ R. Escudero and H. J. T. Smith, J. Appl. Phys. 56, 3271 (1984).

${ }^{26}$ A. S. Deakin and M. A. H. Nerenberg, Phys. Rev. B 25, 1559 (1982).

${ }^{27}$ A. Wolf, J. B. Swift, H. L. Swinney, and J. A. Vastano, Physica D 16, 285 (1985).

${ }^{28}$ J. A. Blackburn, Yang Zhou-jing, S. Vik, H. J. T. Smith, and M. A. H. Nerenberg, Physica D 26, 385 (1987). 